\title{
THE SLICE PRODUCT OF FUNCTION ALGEBRAS ${ }^{1}$
}

\section{LARRY EIFLER}

1. Introduction. A Banach space $E$ is said to have the approximation property (a.p.) if the identity operator on $E$ is in the strong closure of the linear operators on $E$ of finite rank. The disc algebra, $A_{0}$, is the algebra of continuous functions on the closed unit disc which are analytic in the open unit disc. One easily sees that $A_{0}$ has the a.p. by considering Cesaro means. The algebra $A_{0}$ can be looked upon as the closed linear span of a collection of characters on a compact Abelian group or as the uniform closure of polynomials on a compact subset of the plane. Correspondingly, we obtain in $\$ 3$ two generalizations of the result that the disc algebra has the a.p.

In $\$ 2$ we point out the connection between the a.p., slice products, and tensor products. Extension of functions in slice products is examined in the final section.

2. Tensor product and slice product. If $X$ is a topological space, then $C(X)$ denotes the algebra of continuous complex valued functions on $X$. The symbol $\|\square\|$ will always refer to the supremum norm. If $A$ and $B$ are closed linear subspaces of $C(X)$ and $C(Y)$ respectively where $X$ and $Y$ are compact spaces, then the tensor product of $A$ and $B$, denoted by $A \otimes B$, is the closed linear span in $C(X \times Y)$ of functions of the form $f \otimes g$ with $f \in A$ and $g \in B$ where $(f \otimes g)(x, y)=f(x) g(y)$.

Definition 2.1. Let $X$ and $Y$ be topological spaces and let $A$ and $B$ be subsets of $C(X)$ and $C(Y)$ respectively. The slice product of $A$ and $B$, denoted by $A \# B$, is the set

$$
\{F \in C(X \times Y): F(x,-) \in B \text { and } F(-, y) \in A \text { if }(x, y) \in X \times Y\} \text {. }
$$

A Banach space $E$ has the a.p. if and only if for each compact subset $K$ of $E$ and each $\epsilon>0$ there exists a continuous linear operator $T$ mapping $E$ into $E$ such that $T$ has finite rank and $\|x-T(x)\| \leqq \epsilon$ for each $x \in K$. The following result was obtained by Grothendieck $[4$, p. 175] and is an easy consequence of the Hahn-Banach theorem and the Riesz representation theorem.

Proposition 2.2. Suppose $A$ and $B$ are closed linear subspaces of $C(X)$ and $C(Y)$ respectively where $X$ and $Y$ are compact spaces. If $A$ has the a.p., then $A \otimes B=A \# B$.

Received by the editors January 17, 1969.

1 This paper is part of the author's dissertation at Purdue University. 
3. The approximation property for some function algebras. In this section we obtain two generalizations of the result that $A_{0}$ has the a.p. We begin with the case of compact Abelian groups. Let $G$ be a compact Abelian group and $G^{\prime}$ denote the dual group of $G$. A linear subspace $S$ of $C(G)$ is called translation invariant if $f \in S$ and $x \in G$ implies $f_{x} \in S$ where $f_{x}(y)=f(y-x)$. Silov proved [6] that if $S$ is a translation invariant closed linear subspace of $C(G)$, then $S$ equals the closed linear span of $G^{\prime} \cap S$. Using the same techniques as Šilov, one obtains the following

Theorem 3.1. Let $G$ be a compact Abelian group and suppose $S$ is a translation invariant closed linear subspace of $C(G)$. Then $S$ has the a.p.

Proof. Let $K$ be a compact subset of $S$ and let $\epsilon>0$. We may assume $\|f\| \leqq 1$ for each $f \in K$. There exists a neighborhood $U$ of 0 in $G$ such that $\left\|f_{y}-f\right\| \leqq \epsilon / 2$ for each $y \in U$ and $f \in K$ by the uniform continuity of functions in $C(G)$ and the compactness of $K$. Now, there exists $\phi \in C(G)$ such that $\phi$ is nonnegative, $\phi$ vanishes on $G-U$, and $\int \phi d \mu=1$ where $\mu$ denotes the normalized Haar measure on $G$. We have $\|f-f * \phi\| \leqq \epsilon / 2$ for each $f \in K$. By the Peter-Weyl theorem there exists continuous characters $\gamma_{1}, \cdots, \gamma_{n}$ on $G$ and complex numbers $a_{1}, \cdots, a_{n}$ such that

$$
\left\|\phi-\sum_{i=1}^{n} a_{i} \gamma_{i}\right\| \leqq \epsilon / 2
$$

We obtain

$$
\left\|f-f *\left(\sum_{i=1}^{n} a_{i} \gamma_{i}\right)\right\| \leqq \epsilon \quad \text { for each } f \in K .
$$

Define $T: S \rightarrow C(G)$ by $T(f)=f *\left(\sum_{i=1}^{n} a_{i} \gamma_{i}\right)$. Of course, $T$ is a continuous linear operator. Moreover, the range of $T$ is a finite-dimensional subspace of $S$.

EXAMPLE 3.2. Let $T^{2}$ denote the torus and let $\alpha$ be an irrational number. Let $A_{\alpha}$ be the closed subalgebra of $C\left(T^{2}\right)$ generated by the functions $(z, w) \rightarrow z^{n} w^{m}$ where $n+m \alpha \geqq 0$. By theorem $3.1 A_{\alpha}$ is a function algebra on $T^{2}$ and has the a.p.

ExAmple 3.3. Let $A$ denote the closed linear span of $\left\{e^{i \lambda x}: \lambda \geqq 0\right\}$ in the algebra of continuous bounded function on the real line equipped with sup norm. Then $A$ is a function algebra on the Bohr compactification of the real line and has the a.p. by Theorem 3.1.

We now take up our second generalization of the result that the disc algebra has the a.p. Let $X$ be a compact subset of the plane and 
let $R(X)$ denote the uniform closure in $C(X)$ of the rational functions with poles off X. A deep theorem of S. N. Mergelyan [5] states that if the complement of $X$ consists of finitely many components then $R(X)$ equals the set of continuous functions on $X$ which are analytic in the interior of $X$. By a reformulation of Mergelyan's theorem for $R(X)$ and an application of a result of J. Dugundji [3] on the simultaneous extension of continuous functions, we shall show that $R(X)$ has the a.p. if the complement of $X$ consists of finitely many components.

If $\Omega$ is an open subset of the Riemann sphere $S^{2}$, then $H(\Omega)$ denotes the linear space of analytic functions on $\Omega$. We reformulate Mergelyan's theorem as follows:

Theorem 3.4 (Mergelyan). Let $X$ be a compact subset of the plane whose complement consists of finitely many components. Let $D$ be an open disc containing $X$. Let $A(X, D)$ be the set of all continuous functions on the Riemann sphere which vanish off $D$ and are analytic in the interior of $X$. Then for each $\delta>0$ there exists $\Omega=\Omega(X, \delta)$ an open subset of the plane containing $X$ and a linear operator $\psi_{\delta}: A(X, D) \rightarrow H(\Omega)$ such that $\psi_{\delta}$ is of finite rank and

$$
\left\|f-\psi_{\delta}(f)\right\|_{X} \leqq 6,000 \omega_{f}(\delta)
$$

where $\omega_{f}(\delta)=\sup \left\{\left|f(z)-f\left(z^{\prime}\right)\right|:\left|z-z^{\prime}\right|<\delta\right\}$.

We use the above theorem to establish the a.p. for some function algebras.

THEOREM 3.5. If $X$ is a compact subset of the plane whose complement consists of finitely many components, then $R(X)$ has the a.p.

Proof. Let $D$ be an open disc containing $X$. By Dugundji's theorem [3] there exists a linear transformation $\psi$ of $R(X)$ into $A(X, D)$ such that $\psi(f) \mid X=f$ and $\|\psi(f)\|_{D}=\|f\|_{X}$. Let $K$ be a compact subset of $R(X)$ and let $\epsilon>0$. There exists $\delta>0$ such that

$$
\omega_{g}(\delta) \leqq 6,000 \epsilon^{-1} \quad \text { for each } g \in \psi(K)
$$

since $\psi(K)$ is a compact subset of $A(X, D)$. But there exists a linear transformation $\psi_{\delta}$ of $A(X, D)$ into $R(X)$ so that $\psi_{\delta}$ has finite rank and

$$
\left\|g-\psi_{\delta}(g)\right\|_{X} \leqq 6,000 \omega_{g}(\delta) \quad \text { for each } g \in A(X, D) .
$$

Thus

$$
\left\|f-\psi_{\delta}(\psi f)\right\|_{x} \leqq \epsilon \quad \text { for each } f \in K
$$

and so we are done. 
Note. Garnett has pointed out that the recent work of Vitushkin [7] on rational approximation can be used to show that $R(X)$ and $A(X)$ have the a.p. if $X$ is a compact subset of the plane.

COROLlary 3.6. If $A$ is a function algebra with one generator, then $A$ has the a.p.

Proof. There exists a compact subset $X$ of the plane whose complement is connected and such that $A$ is isomorphic and isometric to $R(X)$.

Note. Suppose $A$ and $B$ are closed linear subspaces of $C(X)$ and $C(Y)$ respectively where $X$ and $Y$ are compact spaces. If $A$ and $B$ have the a.p., then $A \otimes B$ has the a.p.

4. Extension of functions in slice products. If $A$ is a function algebra on $X$, then we denote the Silov boundary of $A$ by $\partial_{A}$. The set $\partial_{A}$ is the smallest closed subset of $X$ on which each member of $A$ achieves its maximum modules. If $A$ and $B$ are function algebras, then one easily sees that

$$
\partial_{(A} \otimes_{B)}=\partial_{(A \# B)}=\partial_{A} \times \partial_{B} .
$$

One thus expects invariance of the slice product with respect to the underlying space. Namely, one has the following result due to Birtel [1].

Proposition 4.1. If $A$ and $B$ are function algebras on $X$ and $Y$ respectively, then

$$
(A \# B) \mid\left(\partial_{A} \times \partial_{B}\right)=\left(A \mid \partial_{A}\right) \#\left(B \mid \partial_{B}\right) .
$$

We now take up the problem of extending functions from sets to which the Silov boundary is adherent.

Theorem 4.2. Suppose $A$ and $B$ are function algebras on $X$ and $Y$ respectively. Suppose $X^{\prime} \subset X$ and $Y^{\prime} \subset Y$ such that $\partial_{A}$ is adherent to $X^{\prime}$ and $\partial_{B}$ is adherent to $Y^{\prime}$. If

$F \in C\left(\left(X^{\prime} \cup \partial_{A}\right) \times\left(Y^{\prime} \cup \partial_{B}\right)\right)$ and $F \mid\left(X^{\prime} \times Y^{\prime}\right) \in\left(A \mid X^{\prime}\right) \#\left(B \mid Y^{\prime}\right)$, then there exists a unique function $G$ in $A \# B$ such

$$
F=G \mid\left[\left(X^{\prime} \cup \partial_{A}\right) \times\left(Y^{\prime} \cup \partial_{B}\right)\right] .
$$

Proof. Uniqueness is clear. Existence is established in two parts. We first show that

$$
F \mid\left(\partial_{A} \times \partial_{B}\right) \in\left(A \mid \partial_{A}\right) \#\left(B \mid \partial_{B}\right) .
$$


Fix $x_{0} \in \partial_{A}$. Since $\partial_{B}$ is compact, there exists a sequence $\left(x_{n}\right)_{n=1}^{\infty}$ in $X^{\prime}$ such that

$$
\left\|F\left(x_{n},-\right)-F\left(x_{0},-\right)\right\|_{\partial_{B}} \rightarrow 0 \quad \text { as } n \rightarrow \infty .
$$

We have $F\left(x_{n},-\right)$ belongs to $B \mid\left(Y^{\prime} \cup \partial_{B}\right)$ for $n=1,2, \cdots$ and so $F\left(x_{0},-\right) \mid \partial_{B}$ belongs to $B \mid \partial_{B}$. Similarly, we have that $F\left(-, y_{0}\right) \mid \partial_{A}$ belongs to $A \mid \partial_{A}$ for each $y_{0} \in \partial_{B}$.

Hence, there exists $G$ in $A \# B$ such that

$$
G\left|\left(\partial_{A} \times \partial_{B}\right)=F\right|\left(\partial_{A} \times \partial_{B}\right) \quad \text { by Theorem 4.1. }
$$

We wish to show of course that $G$ equals $F$ on the set $\left(X^{\prime} \cup \partial_{A}\right)$ $\times\left(Y^{\prime} \cup \partial_{B}\right)$. The problem is thus reduced to showing that $F=0$ on $\partial_{A} \times \partial_{B}$ implies $F=0$. But this follows from the continuity of $F$ on $\left(X^{\prime} \cup \partial_{A}\right)\left(Y^{\prime} \cup \partial_{B}\right)$ and the adherence of $\partial_{A}$ and $\partial_{B}$ to $X^{\prime}$ and $Y^{\prime}$ respectively.

We apply the above result to algebras of analytic functions. Let $H_{\infty}(\Omega)$ denote the algebra of bounded analytic functions on $\Omega$ where $\Omega$ is an open subset of the plane and assume that $H_{\infty}(\Omega)$ separates the points of $\Omega$. Let $X$ denote the closure of $\Omega$ in the maximal ideal space of $H_{\infty}(\Omega)$ and let $\hat{H}_{\infty}$ be the Gelfand image of $H_{\infty}(\Omega)$ in $C(X)$. The following result was obtained by Birtel [2] for the case of the open unit disc.

Corollary 4.3. $\hat{H}_{\infty} \# \hat{H}_{\infty}=\{F \in C(X \times X): F \mid(\Omega \times \Omega)$ is bounded and analytic on $\Omega \times \Omega\}$.

Proof. Since the Silov boundary of $\hat{H}_{\infty}$ is adherent to $\Omega$ and since $H_{\infty}(\Omega) \# H_{\infty}(\Omega)$ contains the bounded analytic functions on $\Omega \times \Omega$ we can apply the above theorem.

Let $D$ denote the open unit disc and let $M$ denote the maximal ideal space $H_{\infty}(D)$. One can easily construct bounded analytic functions on $D \times D$ which do not have continuous extensions to $M \times M$. However, each bounded analytic function on $D \times D$ has a bounded continuous extension to $M \times D$. In fact, we have

Proposition 4.4. Suppose $A$ is a function algebra on $X$ and $X^{\prime} \subset X$ such that $\partial_{A}$ is adherent to $X^{\prime}$. If $F$ is a bounded function in $\left(A \mid X^{\prime}\right)$ $\# H_{\infty}(D)$, then there exists a bounded function $G$ in $A \# H_{\infty}(D)$ such that $F=G \mid\left(X^{\prime} \times D\right)$.

Proof. Given $f \in A \mid X^{\prime}$, let $f^{*}$ denote the unique member of $A$ such that $f^{*} \mid X^{\prime}=f$. Define $G: X \times D \rightarrow C$ by

$$
G(x, z)=F(-, z) *(x) .
$$


Since $F$ is bounded, we have that $G$ is bounded. We may assume that $G$ is bounded in modulus by 1 . The crux of the problem is to show that $G$ is continuous on $X \times D$. For this we use that $F(x,-)$ belongs to $H_{\infty}(D)$ as follows. Fix $z_{0} \in D$. For each $z \in D$ we obtain

$$
\begin{aligned}
\left\|G(-, z)-G\left(-, z_{0}\right)\right\| & \leqq \sup \left\{\left|G(x, z)-G\left(x, z_{0}\right)\right|: x \in X^{\prime}\right\} \\
& \leqq 2\left|z-z_{0}\right|\left(1-\left|z_{0}\right|\right)^{-1} .
\end{aligned}
$$

The second inequality follows from the fact that if $z, z_{0} \in D$ and if $f \in H_{\infty}(D)$ then

$$
\left|f(z)-f\left(z_{0}\right)\right| \leqq 2|| f \|_{D}\left|z-z_{0}\right|\left(1-\left|z_{0}\right|\right)^{-1} .
$$

Consequently, $G$ is continuous on $X \times D$. We obtain that $G(x,-)$ belongs to $H_{\infty}(D)$ for each $x \in X$ by applying 4.2 to $G$ restricted to the set

$$
\begin{gathered}
\left(X^{\prime} \cup \partial_{A}\right) \times\{z:|z| \leqq r\} \quad \text { for } 0<r<1 . \\
\text { BibliograPhY }
\end{gathered}
$$

1. F. T. Birtel, "Products of maximal function algebras" in Function algebras, Scott, Foresman \& Co., Glenview, Ill., 1966.

2. - Slice algebras of bounded analytic functions, Math. Scand. 21 (1967), 54-60. 367.

3. J. Dugundji, An extension of Tietze's theorem, Pacific J. Math. 1 (1951), 353-

4. A. Grothendieck, Produits tensoriels topologiques et espaces nucleaires, Mem. Amer. Math. Soc. 16 (1955).

5. S. N. Mergelyan, Uniform approximation of functions of a complex variable, Uspehi Mat. Nauk 5 (1952). no. 2 (48), 31-122; English transl., Amer. Math. Soc. Transl. (1) 3 (1962), 294-391.

6. G. E. Šilov, Homogeneous rings of functions, Uspehi Mat. Nauk 6 (1951), no. 1 (41), 91-137; English transl., Amer. Math. Soc. Transl. (1) 8 (1962), 392-455.

7. L. Zalcman, Analytic capacity and rational approximation, Lecture notes in Math., vol. 50, Springer, Berlin, 1968.

Louisiana State University 